\title{
COOPERACIÓN INTERNACIONAL EN LA LUCHA CONTRA EL TERRORISMO
}

\section{Su Jin Lim}

Aleksandro Palomo Garrido ${ }^{1}$

Facultad de Ciencias Políticas y Sociales

Universidad de Colima, México

http://dx.doi.org/10.5209/NOMA.56875

Resumen: Uno de los aspectos acerca de la cooperación internacional, que más relevancia está tomando en los últimos años es la cooperación internacional en la lucha anti-terrorista. La lucha contra el terrorismo ha pasado a ocupar una importancia creciente en la agenda internacional. Es preciso analizar cómo se está llevando a cabo esta cooperación internacional.

Palabras clave: cooperación internacional, seguridad, terrorismo.

Abstract: One point about international cooperation has become relevant in the last years. It is international cooperation in the fight against terrorism. This fight against terrorism has become increasingly important on the international agenda. It is necessary to analyze how this international cooperation is being developed.

Keywords: International cooperation, security, terrorism.

Uno de los aspectos acerca de la cooperación internacional, que más relevancia está tomando en los últimos años es la cooperación internacional en la lucha anti-terrorista. La lucha contra el terrorismo ha pasado a ocupar una importancia creciente en la agenda internacional. La comprensión de que el fenómeno terrorista es un fenómeno global en un contexto internacional, confirma la necesidad de una cooperación internacional. Es preciso analizar cómo se está llevando a cabo esta cooperación internacional. Evaluar si esta cooperación está siendo efectiva. Considerar si dicha cooperación está respetando los derechos humanos. Así como intentar extraer un diagnóstico acerca de las consecuencias que dicha cooperación está teniendo sobre el sistema político internacional.

\footnotetext{
1 Aleksandro Palomo Garrido y Su Jin Lim son Profesores Titulares de Tiempo Completo en la Facultad de Ciencias Políticas y Sociales de la Universidad de Colima (México). Aleksandro Palomo es además investigador del EMUI. Ambos imparten docencia en la Licenciatura de Relaciones Internacionales de dicha Facultad y en el Doctorado en Ciencias Sociales. Aleksandro Palomo Garrido es Doctor en Ciencia Política por la Universidad Complutense de Madrid (España). Su Jin Lim es Doctora en Geografía Humana por la Universidad Nacional de Seúl (Corea del Sur).
} 


\section{Estado del arte}

Una de las líneas de actuación en la cooperación internacional es la que queda enmarcada dentro de la política de seguridad internacional. En los últimos años la agenda de la política internacional de seguridad ha quedado inundada por el fenómeno del terrorismo. La lucha anti-terrorista ha pasado a protagonizar la política de seguridad internacional. Acerca de este punto y cómo abordarlo hay diferentes puntos de vista. El planteamiento desde el paradigma realista (Aydinli/ Rosenau, 2012) lo contempla como una cooperación puntual entre gobiernos para solucionar un problema concreto. Desde el paradigma idealista (Nye, 2003) se ha hecho hincapié en la formulación de un sistema legalista y regulado por instituciones internacionales. Desde el paradigma neo-marxista (Chomsky, 2010) se contempla como una mera estrategia de los países más poderosos para imponer un ordenamiento mundial favorable a sus intereses. Desde el paradigma constructivista (Buzan, 1998) la cooperación en el ámbito de la seguridad debe abrirse a nuevos causantes períféricos relacionados con los orígenes de la inseguridad.

\section{Planteamiento del problema}

Se entiende por cooperación internacional el conjunto de acciones a través de las cuales se intenta coordinar políticas o unir esfuerzos para poder alcanzar objetivos en el plano internacional. Sin embargo, no hay una única definición de cooperación internacional y mucho menos de su modus operandi. Esto se debe a que el sistema de cooperación internacional se ha vuelto cada vez más complejo, dada la multiplicidad de agentes, objetos, reglas y valores que lo forman. Por ejemplo, los agentes de la cooperación internacional pueden ser personas, corporaciones privadas, gobiernos nacionales, organismos intergubernamentales (Banco Mundial, Naciones Unidas, etc.), gobiernos subnacionales y también organizaciones no gubernamentales (asociaciones, fundaciones, ONGs, etc.) que actuarían tanto en el ámbito nacional como internacional. Además, los ámbitos cubiertos por la cooperación internacional son muy variados.

Uno de los aspectos del problema sería el referido a la cooperación en torno a la política de seguridad internacional. La lucha anti-terrorista ha pasado a protagonizar la política de seguridad internacional en los últimos años. Por tanto, es necesario tomar en cuenta la discusión acerca de la cooperación internacional en el ámbito de la seguridad y valorar las implicaciones derivadas de la lucha anti-terrorista, ya que este hecho está marcando, cada vez más, la agenda internacional. 


\section{El concepto de seguridad}

¿Qué se entiende por seguridad? y ¿̇Cuáles son las amenazas a la seguridad? Al consultar a diferentes autores se encuentran diversas posiciones que de una $u$ otra forma tratan de dar respuesta a los cuestionamientos anteriormente mencionados. El concepto de seguridad ha ido evolucionando a través del tiempo. El concepto ha transitado desde la visión tradicional, que para algunos se queda corta, hasta una visión más contemporánea que ha expandido el ámbito del concepto. A lo largo del texto, revisaremos la concepción de la seguridad de las teorías del realismo, la interdependencia y el constructivismo.

Además del tránsito del concepto referido en las teorías académicas, también se puede observar que la evolución propia del sistema internacional ha influido en la definición del concepto de seguridad. Por ejemplo, podemos distinguir claramente dos períodos diferentes en el sistema internacional de la Guerra Fría y en el sistema internacional posterior de la Globalización. Esto, se debe a que el entorno internacional en cada uno de estos períodos históricos presentó caracteristicas particulares. Estas particularidades concretas han contribuido a que el concepto de seguridad sea entendido de formas diferentes. En concreto, durante la Globalización se han ido introduciendo nuevos sujetos de seguridad para definir el concepto y se ha ampliado la agenda de seguridad, en comparación con el período de la Guerra Fría.

El concepto tradicional de seguridad brota de los autores de la escuela realista, que brindaron a través de sus escritos una forma de analizar y entender el mundo a partir de la concepción del poder. Desde la visión realista, el problema de la seguridad es un problema externo a la nación. Las amenazas a la seguridad de un Estado provienen de otras naciones. En la visión realista, el foco de peligro se ubica en el poder militar que otros Estados poseen y en la utilización de estos recursos para contener la amenaza. El logro de la seguridad se basa en el mantenimiento de los balances militares.

Los realistas parten por reconocer que el sistema internacional es anárquico, es decir, que hay una ausencia de gobierno central, en donde cada uno de los Estados que componen el sistema internacional ejerce su soberanía sobre su territorio y asuntos, siendo los Estados los únicos actores pertinentes del sistema internacional. En este contexto, aparece la autoayuda como la principal motivación que tienen los Estados en el sistema internacional, entendiéndose por autoayuda la necesidad de cada Estado de velar por sus propios intereses, lo cual conlleva al conflicto entre Estados.

Cujabante, 2009. 
La descripción que hacen los realistas del sistema internacional anárquico no deja lugar a dudas. El conflicto entre los Estados determinará un escenario permanente en el entorno mundial. En este escenario conflictivo, los recursos militares cobran gran importancia y tienen gran trascendencia. Como consecuencia, la seguridad de los Estados dependerá del uso de la fuerza. En este sentido, la guerra y la constante posibilidad de guerras hacen que la anarquía del sistema internacional se convierta en una lucha constante por el poder entre los Estados. Así es definido el sistema internacional por Hans Morgenthau (Morgenthau, 1960. p. 48).

El panorama planteado por Hans Morgenthau acerca del sistema internacional permite entender que la seguridad de un Estado, vista desde la concepción realista, está íntimamente relacionada con la capacidad militar que tenga un Estado determinado. En este sentido se podría plantear que, desde el realismo, un Estado estará seguro siempre y cuando tenga la suficiente capacidad militar como para poder responder a políticas de statu quo, imperialistas o de prestigio, llevadas a cabo por actores del sistema internacional, además de contar con los recursos necesarios para ir a la guerra si fuera necesario. Debido a que cualquier Estado podría recurrir al uso de la fuerza, todos los Estados deben estar preparados para hacer lo mismo.

Cujabante, 2009.

En este sentido, si cada uno de los Estados del escenario mundial está buscando afianzar su poder nacional intentando buscar su seguridad, cada uno de los actores del sistema internacional se convierte en una amenaza para la seguridad del otro. Más aún, si se tiene en cuenta que en el sistema internacional los Estados solamente pueden confiar en sus propios esfuerzos por mantener su seguridad, las amenazas a la seguridad se convierten en un problema de supervivencia.

De esta forma, la visión realista reduce las relaciones internacionales a lo más esencial, el manejo de la seguridad y la competencia por los elementos de poder. En este sentido, al analizar el comportamiento de los miembros de cualquier sistema internacional se debe tener en cuenta la distribución del poder y la jerarquía que se establece a partir de la lucha por el poder para garantizar su seguridad.

Cujabante, 2009.

Más moderno que el realismo surgió la teoría de la interdependencia en el sistema internacional. La interdependencia se caracteriza por considerar en sus análisis una multiplicidad de actores. Es decir, que el Estado ya no es el único actor de la escena global, sino que también participan actores no gubernamentales. 
En este sentido, la interdependencia busca redefinir la centralidad otorgada por el realismo al Estado-Nación como unidad de investigación, desagregando sus componentes para lograr una mejor y más completa descripción del sistema internacional. En cuanto a la agenda de relaciones interestatales, ésta va a estar conformada por múltiples temas que no están bajo una jerarquía clara o solida, lo cual significa, entre otras cosas, que la seguridad militar no domina consistentemente la agenda. Muchos temas surgen de la denominada política interna, volviéndose internacionales y haciéndose así cada vez más difusa la diferenciación entre temas internos y externos.

Cujabante, 2009.

La teoría de la interdependencia encajó bien con el escenario de la Globalización, posterior a la Guerra Fría. Las tradicionales amenazas de la Guerra Fría, provenientes de los Estados, dejaron lugar a las llamadas amenazas no tradicionales a la seguridad. Este tipo de amenazas se podían analizar desde la óptica de la interdependencia, en la medida en que éstas se caracterizaban porque la mayoría de ellas no se centraban en el Estado como sujeto de seguridad, sino que emanaban en su mayoría de actores de carácter sub-estatal o trans-estatal. Los principales autores que plantearon esta dinámica determinada por la interdependencia fueron Keohane y Nye (Keohane/ Nye, 1988).

Las amenazas no tradicionales, por su parte, representan un peligro difuso, en la medida en que la fuente de donde provienen es indeterminada, multidimensional, en la medida en que las amenazas pueden provenir de diferentes temas y multidireccional, pues estas amenazas pueden atentar contra la seguridad de actores tanto estatales como no estatales. Además, estas amenazas no pueden ser manejadas solamente por las políticas de defensa tradicionales, es decir, a través de la utilización de los recursos militares, sino que se deben buscar nuevas formas de contrarrestarlas, pues en la mayoría de los casos la utilización de la fuerza no ha sido la herramienta más útil, si tiene en cuenta que estas «nuevas» amenazas presentan características disímiles a las tradicionales. Finalmente, los análisis que se han elaborado sobre este tema sugieren que se deben ampliar los sujetos objeto de seguridad y dejar de considerar al Estado como el único actor cuando de seguridad se trate.

Cujabante, 2009.

Esta nueva serie de nuevas amenazas son el narcotráfico, el terrorismo, la degradación del medio ambiente, la deuda, la pobreza y otras. Estos problemas ponen en riesgo la estabilidad política y democrática. Con la incorporación de estas amenazas al concepto de seguridad surgió toda una tendencia académica a ampliar el concepto de seguridad 
nacional, para ajustarlo al nuevo orden mundial. A los teóricos de la interdependencia se sumaron los teóricos del constructivismo, tales como Barry Buzan, que plantearon la necesidad de ampliar el concepto de seguridad hacia uno que trascienda las limitadas nociones de defensa militar y esté más acorde con el mundo interdependiente de hoy (Buzan, 1991). Buzan sugiere que la seguridad de las colectividades humanas es afectada en cinco sentidos: militar, político, económico, social y del medio ambiente.

Los expansionistas plantean tres argumentos centrales: 1) que el Estado ya no es el único objeto de referencia de la seguridad, sino que se debe incluir a los individuos y colectividades, a la humanidad en su conjunto, la gente en general, la biosfera, entre otros; 2) que cualquier análisis significativo de la seguridad debe considerar la importancia de un rango de amenazas mucho más amplio, entre las que se incluyen aquellas cuyo origen se encuentra en la destrucción ambiental, en la vulnerabilidad económica y en la descomposición de la cohesión social y; 3) que la responsabilidad de proveer seguridad radica no solo en el Estado, sino en instituciones internacionales y en organizaciones no gubernamentales.

Cujabante, 2009.

Con este concepto de seguridad ampliado, la seguridad se convierte en una práctica, una forma específica de enmarcar un problema. De ahí que se pueda denominar a la seguridad como un discurso que se caracteriza por dramatizar un problema, como si éste tuviera prioridad absoluta. Etiquetar un problema como asunto de seguridad significa que el Estado debe desarrollar acciones de urgencia para contrarrestar la amenaza (Waeber, 1995). Esta vía de urgencia para tomar decisiones políticas está siendo utilizada por muchos gobiernos para acelerar la toma de decisiones y en ocasiones sortear los mecanismos democráticos de la toma de decisiones.

En estos casos, surge un problema con la ampliación de la agenda de seguridad, ya que se están securitizando las agendas políticas por parte de los gobiernos. Cualquier tema puede ser justificado por los gobiernos como amenaza a su seguridad y así justificar e implantar políticas antidemocráticas en la búsqueda de la seguridad. Cuando en realidad dichos problemas pueden ser manejados de otra forma.

De ahí que Waever plantee que la securitización es una herramienta con la que cuentan los Estados, pero que se debe saber utilizar, y que así como se puede securitizar la agenda, también se debe tener la capacidad de desecuritizar (desligar determinados asuntos de la agenda de seguridad) algunos temas que pueden ser tratados vía otra tematización.

Cujabante, 2009. 


\section{Cooperación y seguridad}

La interdependencia describe un sistema internacional donde existen múltiples problemas y en el cual las coaliciones son transnacionales y transgubernamentales. En el desarrollo de esta complejidad, el papel potencial de los OIGs (Organismos Inter Gubernamentales) para la negociación política crece considerablemente, pues éstos contribuyen a establecer la agenda internacional y además actúan como catalizadores para la formación de coaliciones. Es en este contexto, donde aparece el concepto de cooperación internacional como una pieza fundamental (Keohane, 1988).

Keohane plantea que se debe distinguir entre cooperación y armonía. Esta última se refiere a una situación en la que las políticas de los actores automáticamente facilitan el logro de los propósitos de otros, mientras que la cooperación requiere que las acciones de individuos $u$ organizaciones, que no se hallan en armonía preexistente, se adecúen mutuamente por medio de un proceso de negociación, al que generalmente se designa «coordinación de políticas». En este sentido, la cooperación se produce cuando los actores adaptan sus conductas a las preferencias presentes o anticipadas de otros, siendo ésta altamente política, pues de algún modo deben alterarse los esquemas de conducta.

Cujabante, 2009.

La interdependencia global, la complejidad de los problemas internacionales y la dificultad creciente en alcanzar un equilibrio de poder satisfactorio, hacen necesaria la cooperación internacional para resolver las complejas situaciones que se presentan. La erosión de la soberanía de los Estados y la multiplicación de los actores transnacionales ha modificado de manera progresiva y en profundidad el sistema internacional y el ordenamiento global. La organización y el funcionamiento de los OIGs deben reformarse para construir un espacio multilateral amplio que permita a todos los actores, públicos y privados, poderosos y débiles, participar en la elaboración de una agenda global.

Deben igualmente desarrollarse mecanismos de prevención y de gestión de crisis, de evaluación, de puesta en práctica y de regulación de las discrepancias, para garantizar una cooperación internacional más legítima y eficaz. Las cumbres mundiales se ha demostrado que no son la solución, pero parecen ser el mejor camino hacia la solución. Las relaciones diplomáticas no tienen el carácter multilateral de las cumbres. Son oscurantistas y alimentan la desconfianza de los no participantes. Es preciso un sistema de cooperación transparente y participativo que no excluya a nadie. 
En estas condiciones, conseguir la paz a nivel global se revela como una tarea particularmente ardua. Los OIGs, como la ONU, se han demostrado ineficaces a la hora de reducir la conflictividad. Su escasa influencia se reduce a negociar los precarios altos del fuego; decretar embargos de armas que son fácilmente burlados por los traficantes de armas; y autorizar el despliegue de fuerzas de intervención internacionales. Sin embargo, su acción queda bloqueada y nunca atacan las verdaderas causas del conflicto.

La ONU se enfrenta a múltiples problemas para cumplir con la tarea del mantenimiento de la paz. No dispone de un ejército propio y debe recurrir a fuerzas multinacionales, compuestas de efectivos prestados por gobiernos voluntarios para cada misión. Por otro lado, el sostenimiento de las misiones depende del apoyo financiero de los Estados. Muchos Estados aprovechan esta circunstancia para presionar en beneficio de sus intereses. Además, las misiones de paz son controvertidas por cuanto avalan el derecho de injerencia y pueden vulnerar la soberanía de los Estados, ya que las fuerzas de intervención se implican en los conflictos sin tener siempre el consentimiento previo del gobierno o de las facciones rivales.

Después de los desastres de Somalia (1992), Bosnia (1992) y Ruanda (1994) la ONU implementó operativos multidimensionales que combinaban imposición del orden con medidas de consolidación de la paz y reconstrucción. Por ello, además de las acciones militares tradicionales, las fuerzas de intervención desarrollan acciones civiles de administración, asuntos políticos, asuntos jurídicos, etc. Con estas medidas, la definición de la paz como la ausencia de enfrentamientos armados ha quedado severamente limitada. El trabajo por la paz incluye la prevención de conflictos. Para ello es necesario: la identificación de las verdaderas causas; la restitución de valores de referencia y convivencia donde el recurso a la violencia quede descartada; reconstruir los vínculos sociales y las comunidades; responsabilizar y juzgar a los inductores e instigadores intelectuales 0 materiales; reconstruir las instituciones públicas y el contrato social.

Cada vez más a menudo se articulan alianzas regionales entre varios Estados para intervenir en un conflicto local. Estas misiones de paz reciben la sanción de la ONU y se ejecutan bajo su mandato. Tal es el caso de Costa de Marfil, Haití, Timor Oriental, Alto Karabaj, Kosovo, etc. Sin embargo, en otros casos, Estados o alianzas de Estados, como la OTAN, están actuando cada vez más a menudo de forma independiente, con o sin el respaldo legal de la ONU. Para actuar de esta forma se basan en la impotencia manifiesta de la ONU para resolver los conflictos, mantener la seguridad mundial y evitar los desastres humanitarios. Esta tendencia acentúa el unilateralismo en la resolución de los conflictos globales y sienta un serio precedente en cuanto a la injerencia en la soberanía de otros Estados. 


\section{Cooperación y terrorismo}

Regresando a la cuestión de la cooperación y la seguridad, vamos a detenernos en el caso concreto del terrorismo y los mecanismos de cooperación internacional que han surgido para hacer frente a esta amenaza a la seguridad. Desde 2001, el terrorismo se ha convertido en la principal amenaza a la seguridad según la ONU (Palomo, 2016). Desde entonces, el terrorismo se ha contemplado como una amenaza de ámbito global que requiere de la cooperación internacional para hacerle frente. En el seno de la ONU se creó el denominado Comité contra el Terrorismo (CCT) basándose en lo dispuesto en las resoluciones 1373 (2001) y 1624 (2005) del Consejo de Seguridad. El CCT trabaja para fortalecer las capacidades de los Estados Miembros de las Naciones Unidas para combatir las actividades terroristas dentro de sus fronteras y en todas las regiones.

El CCT cuenta con la ayuda de la Dirección Ejecutiva del Comité contra el Terrorismo, que aplica las decisiones políticas del Comité, realiza evaluaciones de expertos de cada uno de los Estados Miembros y presta a los países asistencia técnica en la lucha contra el terrorismo (http://www.un.org/es/sc/ctc/, 19/1/17). En cumplimiento de la resolución 2129 (2013) ambos órganos deben coordinar la cooperación internacional. Deben trabajar en estrecha colaboración con los Estados Miembros, en la elaboración de estrategias amplias e integradas contra el terrorismo y la determinación de elementos, normas idóneas y prácticas disponibles.

La resolución 1373 (2001), aprobada de manera unánime el 28 de septiembre de 2001, insta a los Estados Miembros a adoptar una serie de medidas destinadas a reforzar su capacidad jurídica e institucional para combatir las actividades terroristas. En virtud de este objetivo el CCT tiene la función de reclamar que se estreche al máximo la cooperación entre los grupos de expertos; que se intensifique el intercambio ampliado y sistematizado de información; la coordinación de las visitas y la participación en talleres; la asistencia técnica; y las relaciones con organizaciones internacionales, regionales y subregionales.

Además del CCT y de la Dirección Ejecutiva del Comité contra el Terrorismo también existe el Equipo Especial sobre la Ejecución de la Lucha contra el Terrorismo (CTITF). Este equipo fue establecido por el Secretario General en 2005. La función del CTITF es reforzar la coordinación y la coherencia de las actividades de las Naciones Unidas contra el terrorismo. El objetivo principal es maximizar las ventajas comparativas de las aportaciones de cada entidad prestando asistencia a los Estados Miembros en la aplicación de las estrategias (https://www.un.org/counterterrorism/ctitf/es/about-task-force, 19/1/17).

Estos órganos de la ONU organizan la cooperación internacional contra el terrorismo bajo las directrices que recoge la "Estrategia Global de las Naciones Unidas contra el Terrorismo". La Asamblea General de la ONU 
aprobó esta estrategia el 8 de septiembre de 2006 (https://www.un.org/counterterrorism/ctitf/es/un-global-counter-

terrorism-strategy, 19/1/17). Con dicha estrategia se pretende intensificar las iniciativas nacionales, regionales e internacionales de lucha contra el terrorismo. La aprobación de la estrategia cumplió con el compromiso que adquirieron los líderes mundiales en la Cumbre de septiembre de 2005 y se basa en muchos de los elementos propuestos por el Secretario General en su informe del 2 de mayo de 2006 titulado "Unidos contra el Terrorismo: Recomendaciones para una estrategia global contra el terrorismo".

Mediante la adopción de esta estrategia todos los Estados miembros de la ONU acordaron, por primera vez, un enfoque estratégico y operativo común para luchar contra el terrorismo. Con dicha estrategia se pretende organizar la cooperación internacional contra el terrorismo y dar pasos prácticos a nivel individual y colectivo para prevenirlo y combatirlo. Entre ellos se incluyen una amplia gama de medidas que van desde el fortalecimiento de la capacidad de los Estados para afrontar las amenazas terroristas a una mejor coordinación de las actividades del sistema de la ONU relacionadas con la lucha contra el terrorismo.

La Asamblea General de la ONU revisa la estrategia cada dos años, convirtiéndola en un documento vivo y en sintonía con las prioridades de los Estados Miembros en la lucha contra el terrorismo. El último examen de la Estrategia Global de las Naciones Unidas contra el Terrorismo se realizó el 1 de julio de 2016. La Asamblea General estudió el informe del Secretario General relativo a la implementación de la Estrategia durante la última década. También consideró el Plan de Acción para Prevenir el Extremismo Violento, que presentó el Secretario General en enero de 2016 a la Asamblea General. Este órgano aprobó la resolución (A/70/L.50) por consenso (https://www.un.org/counterterrorism/ctitf/es/un-global-counterterrorism-strategy, 19/1/17).

\section{La Estrategia Global de las Naciones Unidas contra el Terrorismo}

La resolución de la Estrategia Global de las Naciones Unidas contra el Terrorismo fue aprobada por la Asamblea General de la ONU y se basa los principios de la Carta de las Naciones Unidas, que incluye las cuestiones relativas a la paz y la seguridad internacionales. En dicho documento, la ONU reconoce que el terrorismo es una de las amenazas más graves para la paz y la seguridad internacionales y admite la necesidad de una "respuesta general, coordinada y coherente contra el terrorismo en los planos nacional, regional e internacional". Por lo que la comunidad internacional debe adoptar las medidas necesarias a fin de aumentar la cooperación para prevenir y combatir el terrorismo. 
Los actos, métodos y prácticas de terrorismo en todas sus formas y manifestaciones constituyen actividades cuyo objeto es la destrucción de los derechos humanos, las libertades fundamentales y la democracia, amenazando la integridad territorial y la seguridad de los Estados y desestabilizando los gobiernos legítimamente constituidos, y que la comunidad internacional debe adoptar las medidas necesarias a fin de aumentar la cooperación para prevenir y combatir el terrorismo.

(https://www.un.org/counterterrorism/ctitf/es/un-global-counterterrorism-strategy 1 19/1/17)

La resolución de la Estrategia Global de las Naciones Unidas contra el Terrorismo urge a los Estados a hacer todo lo posible para llegar a un acuerdo sobre un convenio general contra el terrorismo internacional y concertarlo, incluso resolviendo las cuestiones pendientes relativas a la definición jurídica y el alcance de los actos abarcados por el convenio, a fin de que pueda servir como instrumento eficaz de lucha contra el terrorismo. Además, propone la celebración de una conferencia de alto nivel auspiciada por las Naciones Unidas para formular una respuesta internacional frente al terrorismo en todas sus formas y manifestaciones.

En este sentido, la resolución de la Estrategia Global de las Naciones Unidas contra el Terrorismo contiene una serie de medidas concretas. En primer lugar, presentar la estrategia del documento a una sesión de alto nivel del sexagésimo primer período de sesiones de la Asamblea General. En segundo lugar, revisar la aplicación de la Estrategia después de dos años y examinar sus avances. Admite la posibilidad de actualizarla para responder a los cambios que se hayan producido. Aunque hay que tener en cuenta que muchas de las medidas contenidas en la Estrategia necesitarán tratarse como objetivos a largo plazo.

En tercer lugar, se hace un llamamiento al Secretario General para que haga aportaciones a las futuras deliberaciones de la Asamblea General sobre el examen de la aplicación de la Estrategia y su actualización. En cuarto lugar, alienta a los Estados Miembros y a las organizaciones internacionales, regionales y subregionales pertinentes a apoyar la aplicación de la estrategia. En quinto lugar, anima a las organizaciones no gubernamentales y a la sociedad civil para que se impliquen, según proceda, en determinar cómo incrementar los esfuerzos para aplicar la estrategia.

La base normativa que fundamenta la resolución de la Estrategia Global de las Naciones Unidas contra el Terrorismo proviene de la resolución 49/60 de la Asamblea General (1994); la Declaración complementaria de la Declaración de 1994 sobre medidas para eliminar el terrorismo internacional, que figura en el anexo de la resolución 51/210 de la Asamblea General (1996); y el Documento Final 
de la Cumbre Mundial 2005, en particular la sección relativa al terrorismo. Este último documento es el más reciente y el más influyente.

\section{En el Documento Final de la Cumbre Mundial 2005:}

Los dirigentes de todo el mundo reafirmaron su determinación de apoyartodos los esfuerzos encaminados a preservar la igualdad soberana de todos los Estados, respetar su integridad territorial e independencia política, abstenerse, en sus relaciones internacionales, de la amenaza o el uso de la fuerza en cualquier forma incompatible con los propósitos y principios de las Naciones Unidas, apoyar la solución de controversias por medios pacíficos y de conformidad con los principios de la justicia y el derecho internacional, el derecho a la libre determinación de los pueblos que siguen bajo dominación colonial u ocupación extranjera, la no injerencia en los asuntos internos de los Estados, el respeto de los derechos humanos y las libertades fundamentales, el respeto de la igualdad de derechos de todas las personas sin distinción por motivos de raza, sexo, idioma o religión, la cooperación internacional en la solución de los problemas internacionales de carácter económico, social, cultural o humanitario, y el cumplimiento de buena fe de las obligaciones contraídas en virtud de la Carta.

(https://www.un.org/counterterrorism/ctitf/es/un-global-counterterrorism-strategy, 13/3/17).

El mandato que recogía el Documento Final de la Cumbre Mundial 2005 estipulaba que la Asamblea General desarrollaría sin demora los elementos de una estrategia de lucha contra el terrorismo identificados por el Secretario General, con miras a la aprobación y aplicación de una estrategia que promoviera respuestas generales, coordinadas y coherentes contra el terrorismo en los planos nacional, regional e internacional, y que también tuviera en cuenta las condiciones que propician la propagación del terrorismo. En 2006, el Secretario General elaboró un informe titulado «Unidos contra el terrorismo: recomendaciones para una estrategia mundial de lucha contra el terrorismoı, que fue presentado por el Secretario General a la Asamblea General. Finalmente, la resolución de la Estrategia Global de las Naciones Unidas contra el Terrorismo completaba los requerimientos del mandato del Documento Final de la Cumbre Mundial 2005.

Un aspecto que merece destacarse de la resolución de la Estrategia Global de las Naciones Unidas contra el Terrorismo es la afirmación de que "el desarrollo, la paz, la seguridad y los derechos humanos están interrelacionados y se refuerzan mutuamente". Esta afirmación de la Asamblea General parece acercarnos a un planteamiento del terrorismo más cercano al concepto de seguridad más amplio de todos los que veíamos al principio del texto. La estrategia admite la relación 
directa entre el auge del terrorismo y las condiciones económicas de la población. Además, hace énfasis en el reforzamiento de las leyes internacionales, los derechos humanos y el diálogo entre culturas para obtener éxito en la derrota del terrorismo.

Afirmando la determinación de los Estados Miembros de continuar haciendo todo lo posible para resolver los conflictos, poner fin a la ocupación extranjera, hacer frente a la opresión, erradicar la pobreza, promover el crecimiento económico sostenido, el desarrollo sostenible, la prosperidad mundial, la buena gobernanza, los derechos humanos para todos y el imperio de la ley, mejorar la comprensión intercultural y asegurar el respeto de todas las religiones, valores religiosos, creencias o culturas.

(https://www.un.org/counterterrorism/ctitf/es/un-global-counterterrorism-strategy, 13/3/17).

El Plan de Acción anexo a la Estrategia Global de las Naciones Unidas contra el Terrorismo (A/RES/60/288) exhorta a los gobiernos a adoptar medidas urgentes para prevenir y combatir el terrorismo en todas sus formas y manifestaciones, puesto que constituye una de las amenazas más graves para la paz y la seguridad internacionales. También, exige a los gobiernos que reconozcan que la cooperación internacional y todas las medidas que se adopten para prevenir y combatir el terrorismo deben ajustarse a las obligaciones contraídas en virtud del derecho internacional. La condena al terrorismo debe ser sistemática, inequívoca y firme, independientemente de quién lo cometa y de dónde y con qué propósitos.

Este planteamiento parece prevenir sobre la tentación de los gobiernos a condenar y combatir el terrorismo procedente de determinadas organizaciones, pero no de otras. También, previene sobre la tentación de emplear métodos en la lucha contra el terrorismo que no cumplan con la legalidad internacional y los derechos humanos. Es decir, la aplicación de la "guerra sucia" contra los terroristas. Este planteamiento, a modo de preámbulo, parece obedecer a una observación empírica de unos hechos manifiestos.

En materia de cooperación internacional, el plan de acción hace un llamamiento a que los gobiernos firmen y apliquen los convenios y protocolos internacionales existentes de lucha contra el terrorismo. También, expresa la necesidad de acordar un convenio general sobre el terrorismo internacional. El plan de acción recuerda el compromiso de todos los países de aplicar todas las resoluciones de la Asamblea General sobre medidas para eliminar el terrorismo internacional, así como las resoluciones pertinentes de la Asamblea sobre la protección de los derechos humanos y las libertades fundamentales en la lucha contra el terrorismo. De igual manera, se deben aplicar todas las resoluciones del Consejo de Seguridad relacionadas con el terrorismo 
internacional y cooperar plenamente con los órganos subsidiarios del Consejo de Seguridad.

El plan de acción se desarrolla en torno a cuatro pilares:

1. Hacer frente a las condiciones que propician la propagación del terrorismo.

2. Prevenir y combatir el terrorismo.

3. Desarrollar la capacidad de los Estados Miembros para prevenir y combatir el terrorismo y fortalecer el papel del sistema de las Naciones Unidas al respecto.

4. Garantizar el respeto universal de los derechos humanos y del estado de derecho como pilar fundamental de la lucha contra el terrorismo.

Hacer frente a las condiciones que propician la propagación del terrorismo según el plan de acción de la ONU

En cuanto a las condiciones que propician la propagación del terrorismo, el Plan de Acción anexo a la Estrategia Global de las Naciones Unidas contra el Terrorismo identifica como principales causas a los conflictos prolongados sin resolver; la deshumanización de las víctimas del terrorismo en todas sus formas y manifestaciones; la ausencia del imperio de la ley; las infracciones de los derechos humanos; la discriminación por motivos étnicos, nacionales y religiosos; la exclusión política; la marginación socioeconómica y la falta de buena gobernanza. No obstante, aclara que ninguna de esas condiciones puede excusar ni justificar los actos de terrorismo.

A continuación, recoge ocho puntos para reducir estas causas que condicionan el surgimiento y la propagación del terrorismo.

1. Fortalecer la capacidad de las Naciones Unidas en ámbitos como la prevención de conflictos, la negociación, la mediación, la conciliación, el arreglo judicial, el imperio de la ley y el mantenimiento y la consolidación de la paz, para contribuir a la prevención efectiva y la solución por medios pacíficos de conflictos prolongados sin resolver.

2. Organizar iniciativas y programas auspiciados por las Naciones Unidas para promover el diálogo, la tolerancia y el entendimiento entre civilizaciones, culturas, pueblos y religiones, y promover el respeto mutuo de las religiones, los valores religiosos, las creencias y las culturas, y prevenir su difamación.

3. Promover una cultura de paz, justicia y desarrollo humano, tolerancia étnica, nacional y religiosa, y respeto de todas las religiones, los valores religiosos, las creencias o las culturas estableciendo y promoviendo, según proceda, programas de enseñanza y de sensibilización pública que incluyan a todos los sectores de la sociedad.

4. Adoptar las medidas que sean necesarias y adecuadas, 
dimanadas del derecho internacional, a fin de prohibir por ley la incitación a cometer actos terroristas y prevenir las conductas de esa índole.

5. Reforzar la determinación de asegurar el logro puntual e íntegro de los objetivos y metas de desarrollo para erradicar la pobreza y promover el crecimiento económico sostenido, el desarrollo sostenible y la prosperidad global para todos.

6. Aplicar y reforzar los programas de trabajo en materia de desarrollo e inclusión social, especialmente en lo relativo al desempleo de los jóvenes, ya que podría reducir la marginación y el consiguiente sentimiento de victimización que impulsa el extremismo y el reclutamiento de terroristas.

7. Alentar a Naciones Unidas para que intensifique la cooperación y la asistencia, que ya está prestando en los ámbitos del imperio de la ley, los derechos humanos y la buena gobernanza con el fin de apoyar el desarrollo económico y social sostenido.

8. Estudiar la posibilidad de establecer, a título voluntario, sistemas nacionales de asistencia que atiendan a las necesidades de las víctimas del terrorismo y sus familias y faciliten la normalización de su vida. También, promover la solidaridad internacional en apoyo de las víctimas y fomentar la participación de la sociedad civil en una campaña mundial contra el terrorismo y para su condena.

Prevenir y combatir el terrorismo según el plan de acción de la ONU

En cuanto a las medidas para prevenir y combatir el terrorismo, el Plan de Acción anexo a la Estrategia Global de las Naciones Unidas contra el Terrorismo se centra en impedir el acceso de los terroristas a los medios para realizar sus actividades y que alcancen sus objetivos. También hay que neutralizar los efectos que persiguen con sus actividades. El plan propone 17 puntos para alcanzar esta meta:

En los dos primeros puntos parece traslucirse una preocupación porque los gobiernos puedan recurrir a la "guerra sucia" para combatir el terrorismo o porque puedan respaldar actividades terroristas para lograr sus intereses. En este sentido, el documento exhorta a los gobiernos a no recurrir a estas prácticas como una precondición para prevenir y combatir el terrorismo.

1. No participar ni colaborar en actividades terroristas y adoptar medidas prácticas adecuadas para asegurar que los territorios nacionales no se utilicen como base de organizaciones terroristas.

2. Cooperar plenamente en la lucha contra el terrorismo de conformidad con las obligaciones que nos incumben en virtud del derecho internacional con el fin de localizar, negar refugio y someter a la acción de la justicia, según el principio de extradición o enjuiciamiento, a toda persona que apoye, facilite, participe o trate de 
participar en la financiación, planificación, preparación o comisión de actos terroristas, o proporcione refugio;

El siguiente punto destaca la importancia del respeto a los derechos humanos en el desarrollo del combate al terrorismo. Esta preocupación delata una percepción de que a menudo no se respetan dichos derechos y se actúa contra los terroristas al margen de la ley. Si esto sucede, los gobiernos y el sistema legal pierden su legitimidad.

3. Asegurar que los autores de actos terroristas sean detenidos y enjuiciados o extraditados, de conformidad con las disposiciones pertinentes del derecho nacional e internacional, en particular las normas de derechos humanos, el derecho relativo a los refugiados y el derecho internacional humanitario.

Los siguientes puntos buscan intensificar la cooperación y coordinación de las diferentes autoridades nacionales en materia policial. Resulta llamativo el hecho de que también se haga un llamamiento a intensificar la cooperación internacional, no sólo en lo relativo a los delitos de terrorismo, sino también en lo relativo a otros delitos que puedan estar relacionados con las actividades terroristas. En cierto modo, se reconoce que la cooperación internacional policial todavía no está bien estructurada.

4. Intensificar la cooperación, según proceda, para intercambiar información oportuna y fidedigna respecto de la prevención del terrorismo y la lucha contra él;

5. Intensificar la coordinación y la cooperación entre los Estados en la lucha contra los delitos que puedan guardar relación con el terrorismo, incluido el narcotráfico en todos sus aspectos, el comercio ilícito de armas, en particular de armas pequeñas y armas ligeras, incluidos los sistemas portátiles de defensa antiaérea, el blanqueo de capitales y el contrabando de material nuclear, químico, biológico, radiológico y otros materiales potencialmente letales;

6. Considerar la posibilidad para ser partes sin demora en la "Convención de las Naciones Unidas contra la Delincuencia Organizada Transnacional» y en los tres protocolos que la complementan y de aplicarlos;

7. Adoptar las medidas apropiadas, antes de conceder asilo, para verificar que el solicitante no haya participado en actividades terroristas y, tras la concesión del asilo, para asegurar que la condición de refugiado no se utilice de manera contraria a lo dispuesto en el párrafo 1 de la sección II supra;

8. Alentar a las organizaciones regionales y subregionales competentes a establecer o reforzar mecanismos o centros de lucha contra el terrorismo. En caso de que necesiten cooperación y asistencia a esos efectos, alentamos al Comité contra el Terrorismo y a su Dirección Ejecutiva y, cuando sea compatible con los mandatos existentes, a la 
Oficina de las Naciones Unidas contra la Droga y el Delito y a la Organización Internacional de Policía Criminal, a facilitar dicha cooperación y la prestación de dicha asistencia;

9. Reconocer que podría estudiarse la cuestión de establecer un centro internacional de lucha contra el terrorismo, como parte de los esfuerzos internacionales para intensificar la lucha contra el terrorismo;

10. Alentar a los Estados a aplicar las normas internacionales generales enunciadas en las cuarenta recomendaciones sobre el blanqueo de capitales y las nueve recomendaciones especiales sobre la financiación del terrorismo del Equipo de Acción Financiera, reconociendo que los Estados tal vez necesiten asistencia para aplicarlas;

11. Invitar al sistema de las Naciones Unidas a que, junto con los Estados Miembros, elabore una base de datos única y omnicomprensiva sobre incidentes biológicos, asegurándose de que complemente la base de datos sobre delitos biológicos prevista por la Organización Internacional de Policía Criminal. Alentamos también al Secretario General a actualizar la lista de expertos y laboratorios, así como las directrices y los procedimientos técnicos, que tiene a su disposición para la investigación pronta y eficiente del presunto uso. Señalamos además la importancia dela propuesta del Secretario General de congregar, en el marco de las Naciones Unidas, a los principales interesados en el ámbito de la biotecnología, incluidas las empresas, la comunidad científica, la sociedad civil y los gobiernos, en un programa común cuyo fin sea asegurar que los avances de la biotecnología no se utilicen para el terrorismo ni otros fines delictivos, sino para el bien público, con el debido respeto de las normas internacionales fundamentales sobre los derechos de propiedad intelectual;

El siguiente punto reclama la cooperación internacional para el control de internet. El documento reconoce la importancia de internet como herramienta para la propagación del terrorismo y organización de sus actividades. Para prevenir este hecho, invoca la cooperación y coordinación de los diferentes esfuerzos nacionales. Sin embargo, no especifica qué tipo de medidas recomienda adoptar. Tan sólo establece como una precondición que dichas medidas deben respetar el derecho internacional. Este punto queda un tanto ambiguo por cuanto el control de un medio de comunicación como internet afecta a millones de personas.

12. Cooperar con las Naciones Unidas, teniendo debidamente en cuenta la confidencialidad, respetando los derechos humanos y de conformidad con otras obligaciones dimanadas del derecho internacional, a fin de estudiar formas de:

a. Coordinar esfuerzos, a nivel regional e internacional, para luchar contra el terrorismo en todas sus formas y manifestaciones en Internet; 
b. Utilizar Internet como instrumento para luchar contra la propagación del terrorismo, reconociendo al mismo tiempo que los Estados pueden necesitar asistencia a este respecto.

Los siguientes puntos hacen un llamamiento a intensificar la cooperación internacional en el control de las fronteras nacionales. Se busca establecer un control más exhaustivo del movimiento de personas a través de las fronteras para poder limitar la movilidad de los terroristas. Esto con la intención de obstaculizar sus actividades en una sociedad internacional globalizada. En este sentido ha levantado polémica la elaboración de "listas negras" con individuos sospechosos de colaborar con el terrorismo y que estarían sancionados para viajar. Ya que en muchos casos estos nombres figuran en dichas listas por sospechas, pero no hay pruebas judiciales.

13. Intensificar los esfuerzos a nivel nacional y la cooperación bilateral, subregional, regional e internacional, según proceda, para mejorar los controles fronterizos y aduaneros a fin de prevenir y detectar el desplazamiento de terroristas y prevenir y detectar el tráfico ilícito de, entre otras cosas, armas pequeñas y armas ligeras, municiones y explosivos convencionales, y armas y materiales nucleares, químicos, biológicos o radiológicos, reconociendo al mismo tiempo que los Estados tal vez necesiten asistencia a esos efectos;

14. Alentar al Comité contra el Terrorismo y a su Dirección Ejecutiva a seguir colaborando con los Estados, a petición de éstos, para facilitar la adopción de leyes y medidas administrativas a fin de cumplir las obligaciones relativas al desplazamiento de terroristas, y determinar las mejores prácticas en esa materia, aprovechando, siempre que sea posible, las adoptadas por organizaciones técnicas internacionales, como la Organización de Aviación Civil Internacional, la Organización Mundial de Aduanas y la Organización Internacional de Policía Criminal;

15. Alentar al Comité del Consejo de Seguridad establecido en virtud de la resolución 1267 (1999) a seguir trabajando para aumentar la eficacia de la prohibición de los viajes con arreglo al régimen de sanciones de las Naciones Unidas contra Al-Qaida y los talibanes y personas y entidades asociadas y para asegurar, como cuestión prioritaria, que existan procedimientos imparciales y transparentes para incluir a personas y entidades en sus listas, para retirarlas de ellas y para conceder exenciones por razones humanitarias. A ese respecto, alentamos a los Estados a divulgar información, incluso mediante una distribución amplia de las notificaciones especiales de la Organización Internacional de Policía Criminal y las Naciones Unidas sobre las personas que sean objeto de ese régimen de sanciones;

16. Intensificar las actividades y la cooperación a todos los niveles, según proceda, para mejorar la seguridad de la fabricación y expedición de documentos de identidad y de viaje, y prevenir y detectar su alteración o uso fraudulento, reconociendo al mismo tiempo 
que los Estados tal vez necesiten asistencia para ello. A este respecto, invitamos a la Organización Internacional de Policía Criminal a mejorar su base de datos sobre documentos de viaje robados o extraviados y trataremos de usar plenamente ese instrumento, cuando proceda, en particular mediante el intercambio de la información pertinente;

Los siguientes puntos son un llamamiento a coordinar planes de respuesta en caso de que se cometan actos terroristas y para prevenir dichos actos. De esta manera se pretenden obtener mejores resultados que faciliten una respuesta coordinada y más eficaz. Esta cooperación incluiría la asistencia entre las diferentes autoridades nacionales.

17. Invitar a las Naciones Unidas a mejorar la coordinación de la planificación de la respuesta a los atentados terroristas con armas o materiales nucleares, químicos, biológicos o radiológicos, en particular mediante el examen y el aumento de la eficacia de los mecanismos existentes de coordinación entre organismos para la prestación de asistencia, operaciones de socorro y apoyo a las víctimas, a fin de que todos los Estados puedan recibir una asistencia adecuada. A este respecto, invitamos a la Asamblea General y al Consejo de Seguridad a elaborar directrices sobre la cooperación y la asistencia necesarias en caso de que se cometa un atentado terrorista con armas de destrucción en masa;

18. Intensificar todas las actividades tendientes a mejorar la seguridad y la protección de objetivos particularmente vulnerables, como infraestructura y lugares públicos, así como la respuesta a atentados terroristas y otros desastres, en particular en la esfera de la protección civil, reconociendo al mismo tiempo que los Estados tal vez necesiten asistencia a esos efectos.

Cómo desarrollar la capacidad de los Estados para prevenir y combatir el terrorismo y fortalecer el papel del sistema de la ONU

En cuanto a las acciones para desarrollar la capacidad de los Estados para que puedan prevenir y combatir el terrorismo y fortalecer el papel del sistema de la ONU, el Plan de Acción anexo a la Estrategia Global de las Naciones Unidas contra el Terrorismo contempla una serie de medidas destinadas a hacer más eficaz la acción de los Estados. Estas medidas se recogen en 13 puntos:

En primer lugar, se hace un llamamiento a la obtención de mayores recursos financieros para el combate al terrorismo. Para ello, se alienta a los gobiernos a realizar contribuciones voluntarias para financiar los diferentes proyectos de cooperación. También se propone la búsqueda de nuevas vías de financiación.

1. Alentar a los Estados Miembros a considerar la posibilidad de hacer contribuciones voluntarias a proyectos de las Naciones Unidas de cooperación y asistencia técnica para la lucha contra el terrorismo y a estudiar fuentes adicionales de financiación a ese respecto. Alentamos 
también a las Naciones Unidas a considerar la posibilidad de recurrir al sector privado para conseguir contribuciones para programas de fomento de la capacidad, en particular en las esferas de la seguridad portuaria, marítima y de la aviación civil;

En segundo lugar, se destaca la importancia del intercambio de información entre las organizaciones internacionales y los Estados. Gracias a este intercambio de información, se pueden compartir las mejores prácticas en el combate al terrorismo. Para llevar a cabo este intercambio de información con éxito hay que establecer protocolos y canales de intercambio de la información, así como reuniones de trabajo periódicas.

2. Aprovechar el marco que ofrecen las organizaciones internacionales, regionales y subregionales pertinentes para dar a conocer las mejores prácticas en materia de fortalecimiento de la capacidad de lucha contra el terrorismo, y facilitar sus contribuciones a los esfuerzos de la comunidad internacional en ese ámbito;

3. Considerar la posibilidad de establecer mecanismos apropiados para racionalizar las obligaciones de presentación de informes en materia de lucha contra el terrorismo a que están sujetos los Estados y eliminar la duplicación de solicitudes de informes, teniendo en cuenta y respetando los diferentes mandatos de la Asamblea General, el Consejo de Seguridad y los órganos subsidiarios de éste que se ocupan de la lucha contra el terrorismo;

4. Promover medidas, incluida la celebración de reuniones oficiosas periódicas, para reforzar, según proceda, intercambios más frecuentes de información sobre cooperación y asistencia técnica entre los Estados Miembros, los órganos de las Naciones Unidas que se ocupan de la lucha contra el terrorismo, los organismos especializados competentes, las organizaciones internacionales, regionales y subregionales competentes y la comunidad de donantes, con el fin de fortalecer la capacidad de los Estados para aplicar las resoluciones pertinentes de las Naciones Unidas;

En tercer lugar, se considera la importancia de dotarse de nuevos órganos de cooperación y fortalecer los ya existentes. Esto con la intención de facilitar la cooperación internacional, sobre todo a la hora de coordinar esfuerzos y a la hora de proporcionar asistencia a los Estados que lo requieran. Para ello es necesario el diálogo constante entre las instituciones y el establecimiento de cauces de cooperación permanentes.

5. Acoger con beneplácito la intención del Secretario General de institucionalizar en la Secretaría, dentro de los recursos disponibles, el Equipo Especial para la Lucha contra el Terrorismo a fin de asegurar la coordinación y la coherencia generales de las actividades del sistema de las Naciones Unidas de lucha contra el terrorismo; 
6. Alentar al Comité contra el Terrorismo y a su Dirección Ejecutiva a continuar mejorando la coherencia y la eficiencia de la prestación de asistencia técnica para la lucha contra el terrorismo, en particular intensificando su diálogo con los Estados y las organizaciones internacionales, regionales y subregionales competentes y trabajando estrechamente, incluso intercambiando información, con todas las entidades que prestan asistencia técnica bilateral y multilateral;

7. Alentar a la Oficina de las Naciones Unidas contra la Droga y el Delito, incluída su Subdivisión de Prevención del Terrorismo, a incrementar, en estrecha consulta con el Comité contra el Terrorismo y su Dirección Ejecutiva, la prestación de asistencia técnica a los Estados que la soliciten para facilitar la aplicación de los convenios y protocolos internacionales relacionados con la prevención y represión del terrorismo y las resoluciones pertinentes de las Naciones Unidas;

8. Alentar al Fondo Monetario Internacional, el Banco Mundial, la Oficina de las Naciones Unidas contra la Droga y el Delito y la Organización Internacional de Policía Criminal a aumentar la colaboración con los Estados para ayudarlos a dar pleno cumplimiento a las normas y las obligaciones internacionales relativas a la lucha contra el blanqueo de capitales y la financiación del terrorismo;

9. Alentar al Organismo Internacional de Energía Atómica y la Organización para la Prohibición de las Armas Químicas a proseguir, en el marco de sus mandatos respectivos, sus esfuerzos por ayudar a los Estados a aumentar su capacidad de impedir que los terroristas tengan acceso a materiales nucleares, químicos o radiológicos, velar por la seguridad de las instalaciones conexas y responder eficazmente en caso de que se cometa un atentado con esos materiales;

10. Alentar a la Organización Mundial de la Salud a aumentar su asistencia técnica para ayudar a los Estados a mejorar sus sistemas de salud pública a fin de prevenir los atentados terroristas con armas biológicas y prepararse para ellos;

11. Seguir trabajando en el sistema de las Naciones Unidas para apoyar la reforma y la modernización de los sistemas, instalaciones e instituciones de control de fronteras a escala nacional, regional e internacional;

12. Alentar a la Organización Marítima Internacional, la Organización Mundial de Aduanas y la Organización de Aviación Civil Internacional a reforzar la cooperación entre ellas, colaborar con los Estados para detectar cualesquiera deficiencias nacionales en el ámbito de la seguridad del transporte y prestar asistencia, cuando se solicite, para subsanarlas;

13. Alentar a las Naciones Unidas a colaborar con los Estados Miembros y las organizaciones internacionales, regionales y subregionales competentes para determinar y dar a conocer las 
mejores prácticas de prevención de los atentados terroristas contra objetivos especialmente vulnerables. Invitamos a la Organización Internacional de Policía Criminal a colaborar con el Secretario General para que pueda presentar propuestas a ese respecto. Reconocemos también la importancia de que se emprendan iniciativas de colaboración entre los sectores público y privado en ese ámbito.

Como garantizar el respeto universal de los derechos humanos y del estado de derecho como pilar fundamental de la lucha contra el terrorismo según la ONU

En cuanto a las acciones para garantizar el respeto universal de los derechos humanos y del estado de derecho como pilar fundamental de la lucha contra el terrorismo, el Plan de Acción anexo a la Estrategia Global de las Naciones Unidas contra el Terrorismo considera que las medidas eficaces contra el terrorismo y la protección de los derechos humanos no son objetivos contrapuestos, sino que se complementan y refuerzan mutuamente. Por tanto, se establece que la promoción y la protección de los derechos humanos y el imperio de la ley son elementos esenciales de todos los componentes de la Estrategia contra el terrorismo. El documento contempla 8 puntos en esta dirección:

En primer lugar, se establece un marco legal básico para regular la cuestión de los derechos humanos en relación con el terrorismo.

1. Reafirmar que la resolución 60/158 de la Asamblea General de 16 de diciembre de 2005, constituye el marco básico de la «Protección de los derechos humanos y las libertades fundamentales en la lucha contra el terrorismol;;

2. Reafirmar que los Estados deben asegurar que todas las medidas que se adopten para combatir el terrorismo sean compatibles con las obligaciones dimanadas del derecho internacional, en particular las normas de derechos humanos, el derecho relativo a los refugiados y el derecho internacional humanitario;

En segundo lugar, hace un llamamiento a los Estados a participar en los instrumentos de revisión del cumplimiento de los derechos humanos y a someterse a la actuación de los órganos internacionales competentes.

3. Considerar la posibilidad de pasar a ser partes sin demora en los instrumentos internacionales básicos sobre las normas de derechos humanos, el derecho relativo a los refugiados y el derecho internacional humanitario y de aplicarlos, así como de aceptar la competencia de los órganos internacionales y los órganos regionales pertinentes de vigilancia de los derechos humanos;

En tercer lugar, se pretende promover sólidos sistemas nacionales de justicia que consideren los derechos humanos. Todo ello en armonía con el derecho internacional y el respeto de los derechos humanos y las libertades fundamentales. Al mismo tiempo, las leyes deben tipificar al terrorismo como un delito grave. 
4. Hacer todo lo posible por establecer y mantener un sistema nacional de justicia penal eficaz y basado en el imperio de la ley que asegure, de conformidad con las obligaciones dimanadas del derecho internacional, que se enjuicie a toda persona que participe en la financiación, planificación, preparación o comisión de actos terroristas o apoye tales actos, según el principio de extradición o enjuiciamiento, con el debido respeto de los derechos humanos y las libertades fundamentales, y que se tipifiquen esos actos terroristas como delitos graves en las legislación y los reglamentos nacionales. Reconocemos que los Estados pueden necesitar asistencia para establecer y mantener ese sistema de justicia penal eficaz y basado en el imperio de la ley, y los alentamos a recurrir a la asistencia técnica que prestan entidades como la Oficina de las Naciones Unidas contra la Droga y el Delito;

Por último, se destaca la necesidad de reafirmar el papel de la ONU, como principal organización internacional garante de los derechos humanos y la legalidad internacional. En este sentido, es necesario facilitar y apoyar el trabajo de las organizaciones internacionales encargadas del respeto a los derechos humanos.

5. Reafirmar el importante papel que cabe al sistema de las Naciones Unidas en el fortalecimiento del régimen jurídico internacional mediante la promoción del imperio de la ley, el respeto de los derechos humanos y sistemas eficaces de justicia penal, que constituye en la base fundamental de nuestra lucha común contra el terrorismo;

6. Apoyar al Consejo de Derechos Humanos y contribuir, a medida que se vaya plasmando, a su labor sobre la promoción y la protección de los derechos humanos para todos en la lucha contra el terrorismo;

7. Apoyar el fortalecimiento de la capacidad operacional de la Oficina del Alto Comisionado de las Naciones Unidas para los Derechos Humanos, con especial hincapié en el aumento de las operaciones y la presencia sobre el terreno. La Oficina debería seguir desempeñando una función rectora en el examen de la cuestión de la protección de los derechos humanos en la lucha contra el terrorismo formulando recomendaciones generales sobre las obligaciones de los Estados en materia de derechos humanos y proporcionándoles asistencia y asesoramiento, en particular en cuanto a la difusión de las normas internacionales de derechos humanos entre los organismos nacionales de aplicación de la ley, cuando los Estados lo soliciten;

8. Apoyar la función del Relator Especial sobre la promoción y la protección de los derechos humanos y las libertades fundamentales en la lucha contra el terrorismo. El Relator Especial debe seguir apoyando la labor de los Estados y proporcionando asesoramiento concreto mediante el intercambio de correspondencia con los gobiernos, la realización de visitas a los países, el mantenimiento del enlace con las Naciones Unidas y las organizaciones regionales, y la presentación de informes sobre esas cuestiones. 


\section{Conclusión}

La cooperación internacional en materia de seguridad camina de la mano de la lucha contra el terrorismo. A juzgar por la energía y la generación de documentos que ha elaborado la ONU en relación con la lucha contra el terrorismo, podemos interpretar que ya existe una conciencia entre los gobiernos de que el terrorismo es un problema global que requiere soluciones globales. En concreto, el documento de la ONU sobre la estrategia global contra el terrorismo pretende ser un plan de acción que implica una cooperación internacional entre los diferentes gobiernos. Por tanto, se puede afirmar que la cooperación internacional en el plano de la seguridad camina en la misma dirección que otras cuestiones del ámbito global. Esta dirección apunta hacia el fortalecimiento de los canales de cooperación y coordinación en busca de una mayor efectividad para afrontar un escenario global.

A pesar de ello, es cierto que los asuntos de seguridad conllevan cierto retraso en el estrechamiento de la cooperación, en comparación con otros asuntos como el comercio o las finanzas. Este retraso puede explicarse debido a que la seguridad ha sido un asunto tradicionalmente de ámbito nacional. La seguridad nacional ha sido abordada históricamente por los gobiernos como una cuestión de importancia primaria y de alto secreto. Por tanto, no han existido cauces para compartir información sensible o coordinar operaciones entre los distintos órganos de seguridad de los diferentes gobiernos. El documento de la ONU sobre la estrategia global contra el terrorismo intenta romper esta inercia y propiciar los vínculos entre los agentes implicados.

La lucha contra el terrorismo ha pasado a ocupar un lugar predominante en la agenda internacional cuando el fenómeno ha pisado suelo de Estados Unidos. El 11-S de 2001 marca un claro punto y aparte en el tratamiento de este fenómeno. No podemos aventurar qué dirección puede tomar la cooperación internacional relativa a la seguridad en el futuro. Tampoco podemos asegurar que los cambios producidos sean irreversibles. En un momento como el actual, en el que el proceso de globalización parece atorado se plantean muchas incertidumbres acerca de los canales actuales de cooperación internacional. La cooperación internacional relativa a la seguridad no es una excepción. 


\section{Bibliografía}

- Aydinli, Ersel/ Rosenau, James (2012). Globalization, Security, and the Nation State. SUNY Press.

- Buzan, Barry (1991). People, States and Fear. Lynne Rienner Publishers.

- $\quad$ Buzan, Barry et al. (1998). Security. A New Framework for Analysis. Lynny Rienner Publishers.

- $\quad$ Chomsky, Noam (2010). Estados fallidos. Público.

- Cujabante, Ximena (2009). "La seguridad internacional: evolución de un concepto" en Revista de Relaciones Internacionales, Estrategia y Seguridad, vol. 4, núm. 2. Universidad Militar Nueva Granada.

- Keohane, Robert (1988). "Cooperación y Regímenes Internacionales" en: Keohane, Robert. Después de la Hegemonía. Cooperación y Discordia en la Política Económica Mundial. GEL-RIAL. Buenos Aires

- Keohane, Robert/ Nye, Joseph (1988). Poder e Interdependencia. GEL. Buenos Aires.

- Morgenthau, Hans (1960). Política entre las Naciones. La lucha por el poder y la paz. Editorial Sudamericana.

- $\quad$ Naciones Unidas: http://www.un.org/es/sc/ctc/.

- https://www.un.org/counterterrorism/ctitf/es/about-task-force.

- $\quad$ https://www.un.org/counterterrorism/ctitf/es/un-globalcounter-terrorism-strategy.

- Nye, Joseph S. (2003). La paradoja del poder norteamericano. Santillana.

- Palomo, Aleksandro (2016). "La lucha antiterrorista y el nuevo sistema de seguridad internacional tras el 11 de septiembre: ¿una consecuencia lógica?" en Foro Internacional, LVI-4. Colegio de México.

- Waever, Ole (1995). "Securitization and Desecuritization" en: Lipschutz, Ronnie. On Security. University Press. 